\title{
Episiotomy Scar Endometriosis. Case Presentation
}

\author{
Radu BOTEZATU ${ }^{a, b}$, Ana TURCU-DUMINICA ${ }^{b}$, Anca Marina CIOBANU ${ }^{a, b}$, \\ Nicolae GICA ${ }^{a, b}$, Gheorghe PELTECU ${ }^{a}$, , Anca Maria PANAITESCU ${ }^{a, b}$ \\ a"Carol Davila" University of Medicine and Pharmacy, Bucharest, Romania \\ b"Filantropia" Clinical Hospital, Bucharest, Romania
}

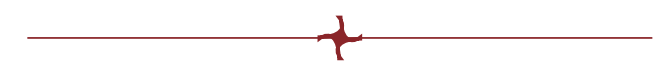

\begin{abstract}
Endometriosis is a frequent gynecological disease among women of reproductive age, a benign condition with an uncertain etiology, but with multiple theories being proposed as main mechanisms of development. Perineal endometriosis is an uncommon condition, with various theories about its pathogenesis including the contiguous propagation of endometrial tissue over the episiotomy perineal scar. A 36-year-old woman, G2 P2, presented with cyclic inflammation and pain in the episiotomy area, with significant impairment of her daily quality of life. Clinical examination revealed a palpable mass in the episiotomy area and a probable clinical diagnosis of perineal scar endometriosis was considered, with further investigation being recommended. The patient was surgically treated by local excision of the perineal mass and the histopathologic examination of the mass confirmed the diagnosis of perineal endometriosis in the episiotomy scar. Although perineal endometriosis remains a rare condition, it should be considered in all patients with an anterior vaginal delivery and a painful perineal mass during menstrual cycle, a wide excision of the affected tissue remaining the best option for a permanent cure.
\end{abstract}

Keywords: endometriosis, perineal endometriosis, scar endometriosis.

\section{INTRODUCTION}

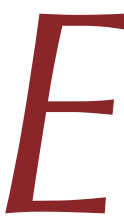
ndometriosis is a benign condition generally found in the pelvic cavity, linked to a hormone-dependent inflammatory mechanism in which endometrial glands and stroma-like tissues develop outside of the uterine cavity (1). Endometriosis can also be found outside the pelvic cavity, involving the umbilicus, abdominal wall, lungs or even vulva or perineum, among other sites (2). According to Andres, a multidisciplinary approach is recommended in order to obtain a better outcome (3). In the reproductive age group, endometriosis affects
$6-10 \%$ of all women, $50-60 \%$ of those referred for pelvic pain and more than $50 \%$ of women diagnosed with infertility (4). Although its etiology remains unknown, the mechanism of retrograde menstruation, introduced for the first time by Sampson in the 1920s, could explain why the Douglas cul-de-sac, ovaries and uterus remain the main sites of the endometriotic lesions (5). As an alternative, according to Watanabe, perineal or abdominal scar endometriosis might be the result of a transplantation seeding mechanism (6). Perineal endometriosis is a rare condition affecting $0.3 \%$ to $1 \%$ of patients, in which endometriotic cells are found in either the skin or subcutaneous

\footnotetext{
Address for correspondence:

Nicolae Gica

Email: gica.nicolae@umfcd.ro
}

Article received on the $6^{\text {th }}$ of October 2021 and accepted for publication on the $22^{\text {nd }}$ of November 2021 
tissue of the vulva and perineum, occasionally involving the perianal sphincteric muscles (7). Perineal endometriosis remains one of the most misdiagnosed conditions in which cyclic pain and inflammation induce a severe impairment of daily activities (8).

\section{CASE REPORT}

46-year-old female G2 P2, five years after her last vaginal delivery which resulted in a repeated right medio-lateral episiotomy, presented with symptoms of cyclic inflammation and pain in the episiotomy area, starting one year prior to presentation, with a significant worsening of her daily quality of life in the last few months. The patient described a significant cyclic pain in the perineum that lasted for 14 days every month, accompanied by inflammation, erythema and a palpable mass in the episiotomy scar area. No bladder or bowel incontinence was reported. Prior to the presentation in the outpatient clinic, the patient was referred to several gynecologists over the last few months, with a clinical diagnosis of perineal granuloma following episiotomy, for which she received antibiotics and anti-inflammatory rectal suppositories. It is important to note that none of the two episiotomies was complicated by local infection. The woman used a hormone-releasing intrauterine device (Levonorgestrel) for contraception that significantly diminished the monthly bleeding, but not the perineal pain or inflammation of the episiotomy scar.

\section{Examination}

The perineal visual examination revealed an episiotomy scar without signs of inflammation or erythema. At the same level, a palpable mass of $3 / 3 \mathrm{~cm}$, firm in consistency and sensitive, was noted. Digital rectal examination exposed a normal sphincter tone and a perineal mass that seemed to involve the rectal wall. A transperineal ultrasound revealed a heterogeneous echogenic mass of $3 / 3 \mathrm{~cm}$ with normal anal sphincter muscles. The patient was referred for a pelvic MRI which showed a well-defined mass of $2.4 \mathrm{~cm}$ in diameter which was located in the right ischioanal fossa, hyperintense $\mathrm{T} 1$, in contact with the right obturator internal muscle, the levator ani muscle and the pudendal vessels (Figure 1). Another stellate perineal mass with a cranio-caudal

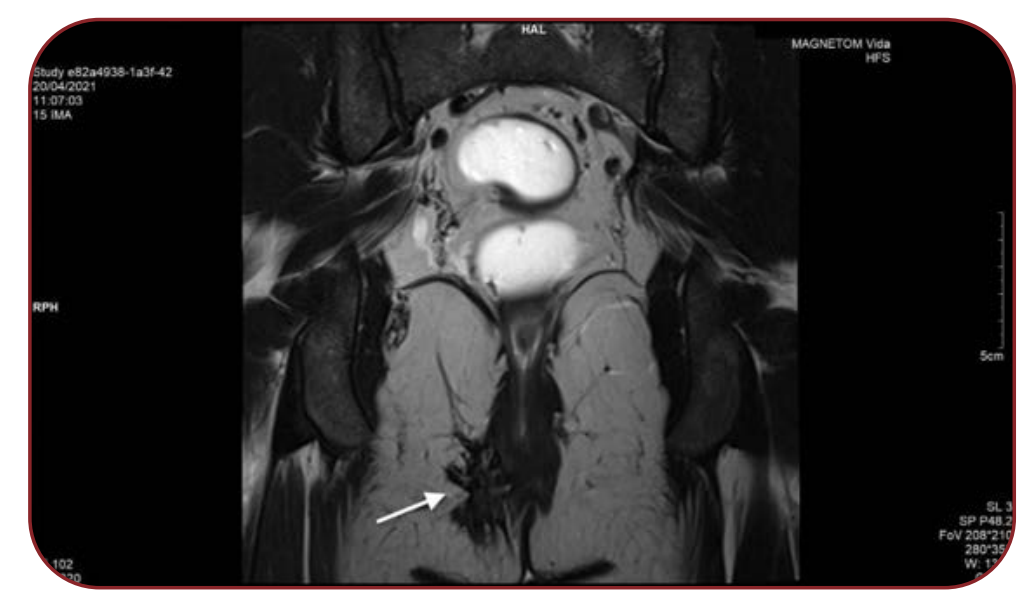

FIGURE 1. Episiotomy scar endometriosis. MRI image, coronal plane

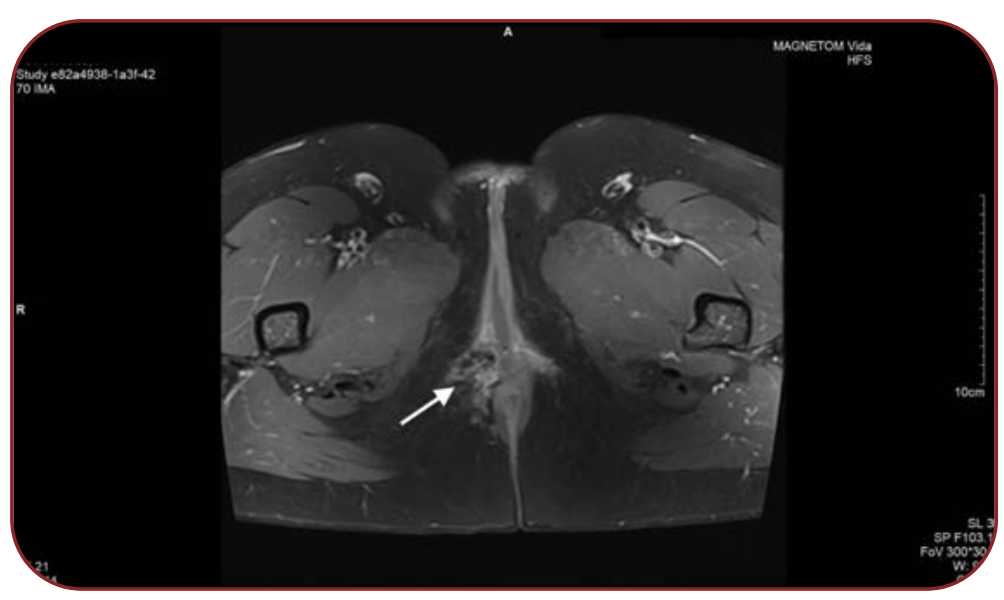

FIGURE 2. Episiotomy scar endometriosis. MRI image, axial plane

diameter of $4 \mathrm{~cm}$ and an axial diameter of $5 / 3.3 \mathrm{~cm}$ was revealed. The lesion did not present the characteristics that could suggest the presence of endometriotic tissue, but it suggested the presence of fibrotic tissue, hypointense $\mathrm{T} 1$, isointense T2 comparative to the skeletal muscle, evolving in the subcutaneous fat tissue (Figure 2). All blood tests were in the normal range.

\section{Management}

The patient was surgically treated by local excision of the perineal mass in the lithotomy position under spinal anesthesia. A well-defined, firm in consistency, perineal mass of $8 \mathrm{~cm}$ in diameter was identified in the deep subcutaneous tissue, without extension into the vaginal or rectal wall but in close contact with them. The external anal sphincter was not involved. The excised tissue was sent for histopathological examination and the patient was discharged on the third postope- 


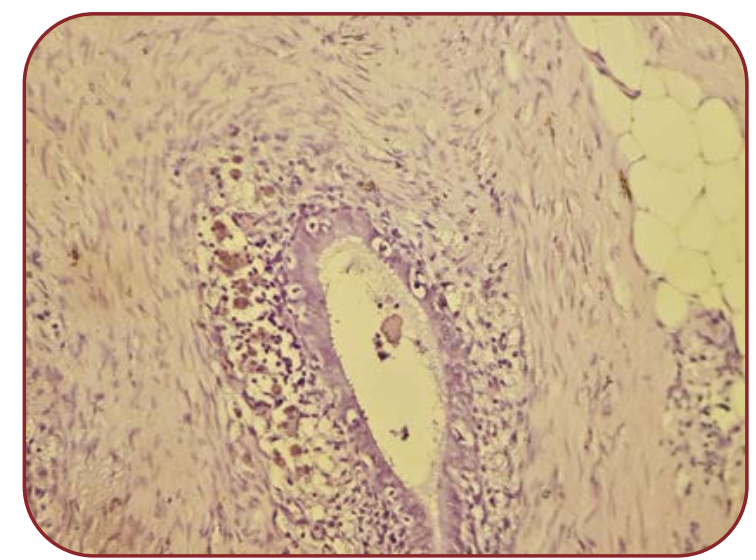

FIGURE 3. Adipose tissue and smooth muscle tissue with endometrial type glands with tubal metaplastic changes embeded in endometrial type stroma with chronic hemorrhage (hemosiderin laden macrophages) (HE, 200x)

rative day. The histopathological examination confirmed the diagnosis of endometriosis (Figure 3). It has also revealed a vascular connective, adipose and muscular tissue, endometrial stroma and hemorrhage areas. The patient experienced a favorable postoperative recovery, with a good perineal healing and the disappearance of pain.

\section{DISCUSSION}

P erineal endometriosis was first reported by Schickele in 1923 (9), while Minvielle and de la Cruz first described it in the anal canal in 1968 (10). Since then, more than 450 eligible studies of perineal endometrioma have been reported. Although vulvo-perineal endometriosis attributes perineal trauma as the main etiology factor, there are other general surgical procedures, including appendicectomy, inguinal hernial repair, laparoscopic cholecystectomy or laparoscopic gastric by-pass, that can cause scar endometriosis (11). However, the literature has also reported 13 cases of perineal endometriosis in patients without any anterior vulvo-vaginal trauma, involving lymphovascular dissemination as the main mechanism of pathogenesis (12). The incidence of vulvo-perineal endometriosis remains low, in a retrospective cohort observational study of 72 patients Nominato and al reporting an incidence of $0.06 \%$ among all patients (13). The median period of time between perineal trauma or surgery and development of symptoms was 30 months, varying from one month to 14 years (14).
According to Maillard, the main symptoms described were vulvar or perineal cyclical pain augmenting during menstruations for $98.2 \%$ of patients, with the remaining $1.8 \%$ experiencing other symptoms such as pain in the labia minora, cyclical bleeding, anal pruritus or infertility (14); $6.1 \%$ of patients have been also diagnosed with pelvic endometriosis, while the average extent of symptomatic disease before medical referrance was one year, varying from two weeks to 20 years (14). The main findings in the clinical examination were vulvo-perineal nodule or mass $(97.5 \%)$, bluish cutaneous lesions (2.1\%) and bilateral polyps of the labia minora (0.4\%) (14).

The precise etiology of endometriosis remains unknown. The two major theories trying to explain the etiology of endometriosis are the metaplasia of coelomic epithelium and the retrograde menstruation which facilitates the implantation of endometrial fragments at the level of the pelvic cavity. Different locations of the disease could not be explained by these two theories.

Perineal endometriosis remains a rare clinical condition. Medical history and clinical signs play a key role in the diagnosis. This is based on three important criteria: woman of reproductive age, past history of vaginal delivery, cyclic pain and inflammation at the level of the episiotomy scar associated with a palpable mass. All these clinical symptoms and signs are seen before the onset of menstrual bleeding (15).

The most adequate explanation for the endometriosis of the episiotomy scar as well as the endometriosis of the abdominal wall scar after cesarean section is the possible transplantation and seeding of endometrial blood and cells during delivery with episiotomy, or cesarean section (15). Simple transplantation is not enough, because a very small number of pregnant patients with episiotomy or cesarean section develop endometriosis. Probably, as in peritoneal endometriosis, there are still unknown individual factors that favor the cells transplant to be accepted.

Treatment is based on surgical excision, a wide local excision with healthy edges reducing the risk of recurrence (16). Surgical treatment may be associated hormonal therapy (gonadotropin-releasing hormone agonists, oral contraceptive pills, progesterons), started before in order to diminish the size of the perineal mass preoperatively. When the anal sphincter is involved, sphincteroplasty is 
required in order to lower the risk of fecal incontinence (17).

\section{CONCLUSION}

The he diagnosis of perineal endometriosis requires an extensive understanding of the patient's medical history and an accurate pelvic examination. Surgery remains the main option for treatment, permanent cure being obtained after complete excision of the affected area. Histopathologic examination is needed for confirmation of the diagnosis, with the presence of endometrial glands and stroma. As reported in the literature and according to our modest experience, removal of affected tissue is recommended, with satisfactory subsequent results. Despite the fact that perineal endometriosis is a rare condition, the diagnosis should be considered in all women with a history of vaginal delivery and cyclical pain and palpable mass in the perineal area, especially during menstruation. The peculiarly of this case was that the patient presented with significant cyclic pain in the perineum that lasted for 14 days every month, with severe impairment in her daily quality of life, and usage of the intrauterine device with Levonorgestrel did not influence the perineal pain or inflammation. The disappearance of pain and improvement in the patient's daily quality of life emphasized the importance of the complete excision of the affected area. Finally, efforts should be made to properly diagnose perineal endometriosis in order to prevent progressive involvement of the anal sphincter.

Conflicts of interest: none declared.

Financial support: none declared.

Authors' contributions: All authors have

contributed equally.

All authors read and approved the final version of the manuscript.

\section{$\boldsymbol{R}_{\text {FFERENCES }}$}

1. Vercellini $P$, Viganò $P$, Somigliana $E$, Fedele L. Endometriosis: pathogenesis and treatment.

Nat Rev Endocrinol 2014;10:261-275. 10.1038/nrendo.2013.255.

2. Davis AC, Goldberg JM. Extrapelvic Endometriosis. Semin Reprod Med 2017;35:98-101. doi: 10.1055/s-0036-1597122.

Epub 2016 Dec 19. PMID: 27992931.

3. Andres MP, Arcoverde FVL, Souza CCC, et al. Extrapelvic Endometriosis: A Systematic Review.

J Minim Invasive Gynecol 2020;27:373-389. doi: 10.1016/j.jmig.2019.10.004. Epub 2019 Oct 13. PMID: 31618674.

4. Giudice LC. Clinical practice.

Endometriosis. N Engl J Med 2010;362:2389-2398. doi:10.1056/NEJMcp1000274.

5. Maeda $K$, Hashimoto $M$, Katai $H$, et al. Endometriosis in episiotomy scar involving anal sphincter.

Journal of the Japan Society of Colo-Proctology 1993;46:891-894.

https://doi.org/10.3862/

jcoloproctology.46.891.

6. Watanabe M, Kamiyama G, Yamazaki K, et al. Anal endosonography in the diagnosis and management of perianal endometriosis: report of a case. Surg Today 2003;33:630-632.

\subsection{7/s00595-003-2545-z.}

7. Chen N, Zhu L, Lang J, et al. The clinical features and management of perineal endometriosis with anal sphincter involvement: a clinical analysis of 31 cases.

Hum Reprod 2012;27:1624-1627. 10.1093/humrep/des067.

8. Dougherty LS, Hull T. Perineal endometriosis with anal sphincter involvement: report of a case. Dis Colon Rectum 2000;43:1157-1160. 10.1007/BF02236565.

9. Schickele: Bull. et Mem. 11e la Soc. Anat. de Paris 20: 601, 1923.

10. González-Longoria G, Mejía-Ovalle RR, Salinas-Aragón E, et al. Endometrioma perianal involucrando esfínter anal externo: reporte de un caso [Perineal endometriosis with anal external sphincter involvement: a case-report]. Rev Gastroenterol Mex 2011;76:173-177. Spanish.

11. Cinardi N, Franco S, Centonze D, Giannone G. Perineal scar endometriosis ten years after Miles' procedure for rectal cancer: Case report and review of the literature. Int J Surg Case Rep 2011;2:150-153.

12. Zhu L, Lang J, Wong $F$, Guo L. Perineal endometriosis without perineal trauma: a case report.
Chin Med J (Engl) 2003;116:639-640.

13. Nominato NS, Prates LFVS, Lauar I, et al. Endometriose de cicatriz cirúrgica: estudo retrospectivo de 72 casos Scar endometriosis: a retrospective study of 72 patients.

Rev Bras Ginecol Obstet 2007;29:403-407.

14. Maillard C, Cherif Alami Z, Squifflet JL, et al. Diagnosis and Treatment of Vulvo-Perineal Endometriosis: A Systematic Review. Front Surg 2021;11;8:637180.

15. Zhu L, Lang J, Wang $H$, et al. Presentation and management of perineal endometriosis. Int J Gynaecol Obstet 2009;105:230-232. doi: 10.1016/j.ijgo.2009.01.022. Epub 2009 Mar 28. PMID: 19329117.

16. Odobasic A, Pasic A, Iljazovic-Latifagic E, et al. Perineal endometriosis: a case report and review of the literature.

Tech Coloproctol. 2010;14 Suppl 1:S25-S27. doi: 10.1007/s10151-010-0642-8. PMID: 20862505.

17. Tapia A, Rahmer A, Oddó $\mathrm{H}$, et al. Endometriosis perineal. Experiencia clínica en 8 pacientes (Perineal endometriosis. Clinical experience with 8 patients).

Rev Med Chil 1995;122:537-541. Spanish. PMID: 7724894. 